\title{
Developing a dynamic virtual stimulation protocol to induce linear egomotion during orthostatic posture control test
}

\author{
Paulo José Guimarães Da-Silva*, Maurício Cagy, Antonio Fernando Catelli Infantosi
}

\begin{abstract}
Introduction: In this work, the effect of a dynamic visual stimulation (DS) protocol was used to induce egomotion, the center of pressure (COP) displacement response. Methods: DS was developed concerning the scenario structure (chessboard-pattern floor and furniture) and luminance. To move the scenario in a discrete forward (or backward) direction, the furniture is expanded (or reduced) and the black and white background is reversed during floor translation while the luminance is increased (or reduced) by steps of $2 \mathrm{~cd} / \mathrm{m}^{2}$. This protocol was evaluated using COP signals from 29 healthy volunteers: standing on a force platform observing the virtual scene $\left(1.72 \times 1.16 \mathrm{~m}\right.$ ) projected $1 \mathrm{~m}$ ahead (visual incidence angle: $\theta \mathrm{l}=81.4^{\circ}$ and $\theta \mathrm{v}=60.2^{\circ}$ ), which moves with constant velocity $(2 \mathrm{~m} / \mathrm{s})$ during $250 \mathrm{~ms}$. A set of $100 \mathrm{DS}$ was applied in random order, interspersed by a $10 \mathrm{~s}$ of static scene. Results: The Tukey post-hoc test $(p<0.001)$ indicated egomotion in the same direction of DS. COP displacement increased over stimulation $(8.4 \pm 1.7$ to $22.6 \pm 5.3 \mathrm{~mm})$, as well as time to recover stability (4.1 \pm 0.4 to $7.2 \pm 0.6 \mathrm{~s}$ ). The peak of egomotion during $\mathrm{DS}_{\mathrm{F}}$ occurred $200 \mathrm{~ms}$ after $\mathrm{DS}_{\mathrm{B}}($ Wilcoxon, $p=0.002)$. Conclusion: The dynamic configuration of this protocol establishes virtual flow effects of linear egomotion dependent on the direction of the dynamic visual stimulation. This finding indicates the potential application of the proposed virtual dynamic stimulation protocol to investigate the cortical visual evoked response in postural control studies.
\end{abstract}

Keywords: Center of pressure, Dynamic visual stimulation, Egomotion, Postural control, Virtual reality, Visual optic flow.

\section{Introduction}

Virtual reality technologies have been used in postural research and neurorehabilitation to compose a dynamic visual stimulation scene (DS) during balance control test (Akizuki et al., 2005; Darekar et al. 2015; Keshner and Kenyon, 2004; Streepey et al., 2007; Wang et al., 2010). This technique has the advantage of deploying specific scenarios, similar to the real world, to be applied simultaneously with a force platform in order to investigate body sway response due to subject's self-motion illusion, also called egomotion (Akizuki et al., 2005; Guerraz and Bronstein, 2008; Keshner et al., Slobounov et al., 2006; 2006; Streepey et al., 2007; Tossavainen et al., 2003). Nevertheless, this evoked postural response is dependent on the visual motion processing and the central brain integration of the environment information with the proprioceptive and vestibular system (Akizuki et al., 2005; Guerraz and Bronstein, 2008; Keshner et al., 2004).

In real situation, the body displacement produces motion of the visual scene at the retina (Berthoz et al., 1975; 1979; Gibson, 1950; 1954; Mestre, 1992; Paulus et al., 1984; 1989; Warren 1995; Warren and Hannon, 1988). This displacement generates a optic flow that specifies the kinematic properties of body sway and also the three-dimensional (3-D) layout of the environment (Guerraz and Bronstein, 2008; Guerraz et al., 2001a; 2001b; O'Connor et al., 2008; Pretto et al., 2009). However, in the moving room paradigm, the egomotion may be enhanced by the properties of the surround motion that can provide spatial reference for postural response (Berthoz et al., 1975; Dijkstra et al., 1994; Gibson, 1950; Lestienne et al., 1977; Masson et al., 1995; Paulus et al., 1989; Stoffregen, 1985; 1986; Van Asten et al., 1988). On this basis, the optic flow from the moving virtual environment can be manipulated to evoke egomotion (Dokka et al. 2009; Keshner and Kenyon, 2000; O'Connor et al., 2008; Pretto et al., 2009; Seno et al., 2009; 2010; Wang et al., 2010).

An important source of information for egomotion relies mostly on the visual properties. Hence, it is affected by the visual incidence angle, distance from the objects, motion velocity, luminance and contrast content of the scenario (Dijkstra et al., 1992; Masson et al., 1994; Seno et al., 2009; 2010; Streepey et al., 2007). Moreover, linear egomotion can be generated by object movement within the 
peripheral visual field and by the floor translation parallel to the anterior-posterior $(\mathrm{A} / \mathrm{P})$ axis of the human body (Berthoz et al., 1975; 1979; Da-Silva et al., 2015; Gibson, 1950; Haibach et al., 2008; Warren and Hannon, 1988), changing COP position at the same direction of the dynamic visual stimulation. Therefore, there is a direct relationship between the direction (and velocity) of visual optic flow and the orientation (and amplitude) of COP displacement (Dokka et al., 2009; 2010; Masson et al., 1995; Reed-Jones et al., 2008; Tossavainen et al., 2003; Wang et al., 2010).

Previous studies of orthostatic postural control and visual optic flow have been designed from many different perspectives. One of them consists in determining whether a particular visual property is used to control postural sway (Andersen and Dyre, 1989; Kuno et al., 1999; Van Asten et al., 1988) or to describe the postural feedback loop (Day and Guerraz, 2007). Moreover, the virtual moving environment has been used to assess time to recover stability and fall risk (Haibach et al., 2008). However, in the dynamic scene stimulation, not all visual information can be used to induce egomotion (Fushiki et al., 2005; O'Connor et al., 2008; Pretto et al., 2009; Wang et al., 2010). Instead, it was stated that the visual optic flow structure as a whole determines the orientation and the magnitude of the postural reaction to the motion of the environment. The optic flow pattern corresponds to the optic motion of environmental elements which indicate the magnitude, velocity and direction of its displacement. The velocity contains information about distance along the object (motion perspective), whereas the direction provides information about self-motion (visual kinesthesis) (Gibson, 1950; Lishman and Lee, 1973). In particular, the focus of scene expansion corresponds to the current direction of egomotion (representing an anterior translation of the body). Thus, the dynamic structure of the optic flow (the 3-D layout) over a stationary observer can be the functional element to induce egomotion during stabilometric test.

Alternatively, since the visually evoked postural responses are dependent of the optic flow (Dokka et al. 2009; Pretto et al., 2009; Seno et al., 2009; 2010;
Wang et al., 2010), the dynamic visual protocol could be designed from the perspective to understand the cortical interaction underlying the visual motion signal processing for postural control. Thus, to investigate this motion-related visual evoked potential, it is important to ensure that the dynamic virtual stimulation protocol generates egomotion. For instance, the present work aims to assess the dynamic virtual stimulation protocol to be applied during stabilometric test: orthostatic posture on a force platform. The visual stimulation was carried out by developing a virtual environmental structure concerning scenario displacement, visual image velocity and incidence angle, distance from the screen and luminance. In this protocol, the reversing black and white background during floor translation parallel to the ground plane was applied simultaneously with the lateral walls and furniture movements while luminance was changed to generate bi-dimensional optic flow stimulation as a tunnel pattern. This dynamic configuration establishes an advantage to generate a virtual flow field that reflects the natural vision and the physiological properties of the body sway perturbation. Indeed, it was employed to evoke illusion of motion in an opposite direction of the dynamic visual stimulation. To this end, we measured and compared the A/P COP displacement during dynamic (forward and backward) and static scenarios, synchronized at motion-onset of the exhibiting dynamic scenes.

\section{Methods}

\section{Dynamic virtual stimulation protocol}

The virtual scenario reproduces an $8.0 \mathrm{~m}$ wide by $3.0 \mathrm{~m}$ high by $12.0 \mathrm{~m}$ deep room developed using IDE Delphi and API OpenGL (Graphics Library). The room consists of a chessboard-pattern floor (similar to pattern-reversal stimulation), with walls and ceiling designed with distinct texture (Figure 1). Furniture can be added and allocated at any position of the room. Hence, the virtual scenario can be composed with different configurations, setting out the dynamic stimulation in the center and/or periphery of the visual

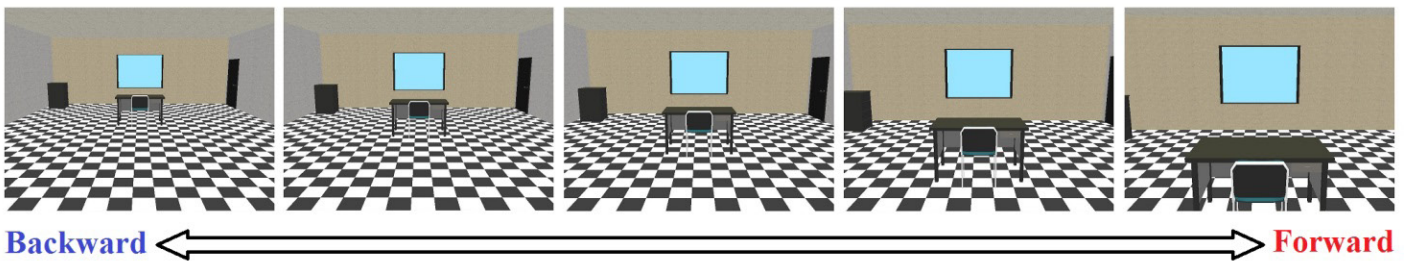

Figure 1. Virtual scenario: chessboard-pattern floor with a table and chair in the center of the screen. Five different views from the most backward to the far forward scenario with displacement in steps of $50 \mathrm{~cm}$ and its corresponding luminance in steps of $2 \mathrm{~cd} / \mathrm{m}^{2} \mathrm{starting}$ in $31 \mathrm{~cd} / \mathrm{m}^{2}$. 
field. As a default, the table and chair was placed in the center of the visual field, while door and drawer in the periphery.

In order to carry out the dynamic properties of the visual stimulation protocol, the furniture is expanded or reduced, while the floor, walls and ceiling are moved in parallel direction with a constant displacement and velocity. Therefore, the virtual scenario can move in forward $\left(\mathrm{DS}_{\mathrm{F}}\right)$ or backward direction $\left(\mathrm{DS}_{\mathrm{B}}\right)$ in a frame rate of $60 \mathrm{~Hz}$. Furthermore, the luminance was changed for each DS scene (Figure 1) by steps of $2 \mathrm{~cd} / \mathrm{m}^{2}$, ranging from 31 to $39 \mathrm{~cd} / \mathrm{m}^{2}$ and increasing in forward direction. The DS sequence was randomly applied and interspersed by a Static Scene (SS) at the final position of the exhibiting DS (the initial position of the next DS). Consequently, the subject could not previously predict the DS direction. Moreover, the amount of DS to be applied, the displacement of the scenario and the time duration of exhibiting DS and SS scenes can be set by configuration. Additionally, the DS stimuli were codified by pulses with specific code numbers for forward and backward displacement (synchronized by the onset of exhibiting DS scene). This sequence of pulses generates a trigger signal to be used during signal processing.

\section{DS during stabilometric test}

The DS protocol was evaluated using the stabilometric signals from 29 healthy subjects (aging from 20 to 42 years), with normal or corrected-o-normal vision and no history of equilibrium disorder, as reported by Da-Silva et al. (2015). All participants previously signed an informed consent form, as approved by the ethics committee of clinical research of IESC/UFRJ - Instituto de Estudos em Saúde Coletiva/Universidade Federal do Rio de Janeiro: 100/2011 (Rio de Janeiro, Brazil).

The stabilometric tests were performed under controlled environmental condition, with the subject in orthostatic posture, quietly standing on a force platform located $1 \mathrm{~m}$ ahead of a white wall. The first test was acquired without virtual scenario stimulation, with the subject just observing the white wall during 5 min (condition denotes as WW). The DS protocol trial was performed with the virtual environment projected on the white wall: displayed with dimension of $1.72 \times 1.16 \mathrm{~m}$ and centered at the subjects vision line as reference. For this evaluation, the DS configuration was set up to generate a moving scene with constant velocity, $V i=2 \mathrm{~m} / \mathrm{s}$, which is considered as one that elicits more postural changes due to illusory egomotion (Berthoz et al. 1975; 1979; Dokka et al., 2009). Thus, the amplitude of each virtual scene motion was $50 \mathrm{~cm}$; displayed during $250 \mathrm{~ms}$. A set of 100 DS stimuli (50 to each direction) was applied in random order, interspersed by $10 \mathrm{~s}$ of SS to allow the balance reestablishment and thus to avoid the risk of falling. This set up configuration generates a dynamic effect of discrete forward and backward stimulation to induce an illusion of egomotion, i.e. self-motion in an opposite direction of the dynamic scenario. All participants were instructed to maintain the orthostatic posture while looking straight ahead at the scene, keeping the gaze at the virtual chair. Moreover, at the final of the experiment, the participants were asked about their perception of self-motion.

\section{Visual optic flow structures}

The visual optic flow was composed based on the visual incidence angle $(\theta)$, the distance between the eyes and the screen $(H)$ and the images velocity (Vi) (Berthoz et al., 1975). Considering the width and height of the image size $(S)$ as $w=1.72 \mathrm{~m}$ and $h=1.16 \mathrm{~m}, H=1.0 \mathrm{~m}$ and $V i=2.0 \mathrm{~m} / \mathrm{s}$, the instantaneous two-dimensional velocity field was estimated as:

$$
\frac{d \theta \bullet}{d t}=\frac{V i}{H} \times \sin ^{2} \theta \bullet
$$

where, $\theta \bullet=2 \times \arctan (S / 2 H)$ is the lateral $\left(-40.7^{\circ} \leq \theta 1\right.$ $\left.\leq 40.7^{\circ}\right)$ or vertical $\left(-30.1^{\circ} \leq \theta \mathrm{v} \leq 30.1^{\circ}\right)$ components of the visual incidence angle obtained for the respective width and height of the image size $(S)$. As describe by Da-Silva et al. (2015), it generated visual optic flow stimulation as a tunnel pattern, with optic flow closer to $0 \% \mathrm{~s}$ in the central vision. Forwards stimulation $\left(\mathrm{DS}_{\mathrm{F}}\right)$ increases the optic flow of the image up to $4 \%$ in both periphery and ground plane of the visual field. Otherwise, backwards $\left(\mathrm{DS}_{\mathrm{B}}\right)$, the optic flow decreases.

\section{The A/P COP signal analysis}

The COP signal was acquired using a square portable force platform $\left(0.16 \mathrm{~m}^{2}\right)$ composed with four load cells (gain: 600x), digitized at $400 \mathrm{~Hz}$ (resolution: 16 bits) with a $60 \mathrm{~Hz}$ digital notch filter, and stored in a hard disk for offline signal processing (Matlab v. 7.6.0 - The Mathworks, USA). Only the COP displacement in the anterior-posterior direction (A/P stabilogram) was analyzed in this evaluation.

The A/P COP signal was low-pass filtered (cut-off: $7 \mathrm{~Hz}$ ) by applying a $2^{\text {nd }}$ order Butterworth with null phase (Da Silva et al., 2012). In the graphs, a positive $\mathrm{A} / \mathrm{P}$ COP displacement means a forward displacement of the subject's COP. Based on the trigger signal, the $\mathrm{A} / \mathrm{P}$ COP signal of each $\mathrm{DS}_{\mathrm{F}}$ was identified and segmented into $M=50$ epochs of $13 \mathrm{~s}$ duration ( -3 to $10 \mathrm{~s}$ ), synchronized by the onset of the $\mathrm{DS}_{\mathrm{F}}$ $(t=0)$ and including $3 \mathrm{~s}$ of SS immediately preceding it. The postural stability just before DS, the changes 
in the $\mathrm{A} / \mathrm{P} \mathrm{COP}$ position over the following time and the time to recover stability after egomotion were assessed by applying the multiple-comparison test (for more details, see Da-Silva et al., 2015).

In summary, the linear egomotion was considered when the distribution of the A/P COP position statistically differed (one-way ANOVA, $\alpha=0.05$ ) from those preceding $\mathrm{DS}_{\mathrm{F}}$ and the COP displacement estimated as the difference of this position identified by the Tukey test $(\alpha=0.05)$ and the position preceding the dynamic scene. Considering only the COP signals with effect of linear egomotion, the time to recover stability was assessed by applying the Tukey test $(\alpha=0.05)$ to identify the consecutive COP segments distributions after $\mathrm{DS}_{\mathrm{F}}$ peak without significant differences. It was established as the instant time of the first initial set that considers COP stability. Finally, those A/P COP signals with effect of linear egomotion were coherently averaged. The same procedure was used for the $\mathrm{DS}_{\mathrm{B}}$ stimulation.

\section{Results}

Figure 2 depicts the A/P COP displacement without virtual stimulation (WW condition) and in response to linear egomotion by applying $\mathrm{DS}_{\mathrm{F}}(\mathrm{red})$ and $\mathrm{DS}_{\mathrm{B}}$ (blue) stimulation. It can be noted that, during WW condition (Figure 2a), the A/P COP displacement oscillates around zero, indicating postural stability over the time. Regardless $\mathrm{DS}_{\mathrm{F}}$ and $\mathrm{DS}_{\mathrm{B}}$ (Figure 2b), no significant change was observed between $\mathrm{COP}$ position during SS before DS (ANOVA, $p>0.8$ ), indicating postural stability just preceding DS. For both $\mathrm{DS}_{\mathrm{F}}$ and $\mathrm{DS}_{\mathrm{B}}$, COP sway increases with the sequence of stimulation, presenting more variability at the final stimuli. Moreover, the postural response after $\mathrm{DS}_{\mathrm{F}}$ (Tukey post-hoc test, $p<0.001$ ), i.e. the negative peak of the stabilogram signals (indicated by the arrow in Figure 2b), and the instant time to recover stability (Tukey post-hoc test, $p>0.6$ ) are time-shifted along the stimulation. Similar result is observed by applying $\mathrm{DS}_{\mathrm{B}}$ (positive peak).

For the same subject, linear egomotion was observed in $63 \%$ of DS. The coherently averaged A/P COP stabilogram during $\mathrm{DS}_{\mathrm{F}}$ (33 stimuli, red line in Figure 3) evidenced postural instability in the posterior direction of the body axes with mean displacement of $-12.3 \mathrm{~mm}$. On the other hand, during $\mathrm{DS}_{\mathrm{B}}$ (31 stimuli, blue line), the instability occurred at the anterior direction with mean displacement of $12.1 \mathrm{~mm}$. Those displacements indicate that the optic flow stimulation as a tunnel pattern induced linear

WW Condition

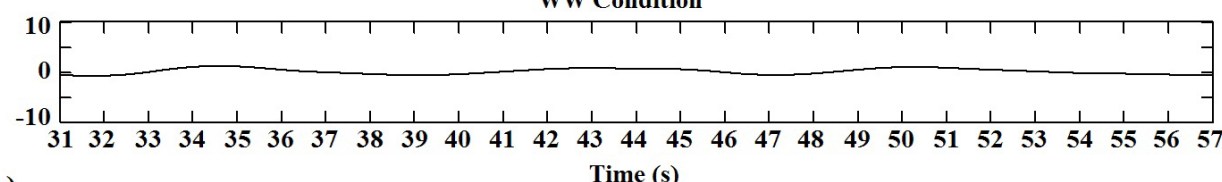

a)

Time (s)
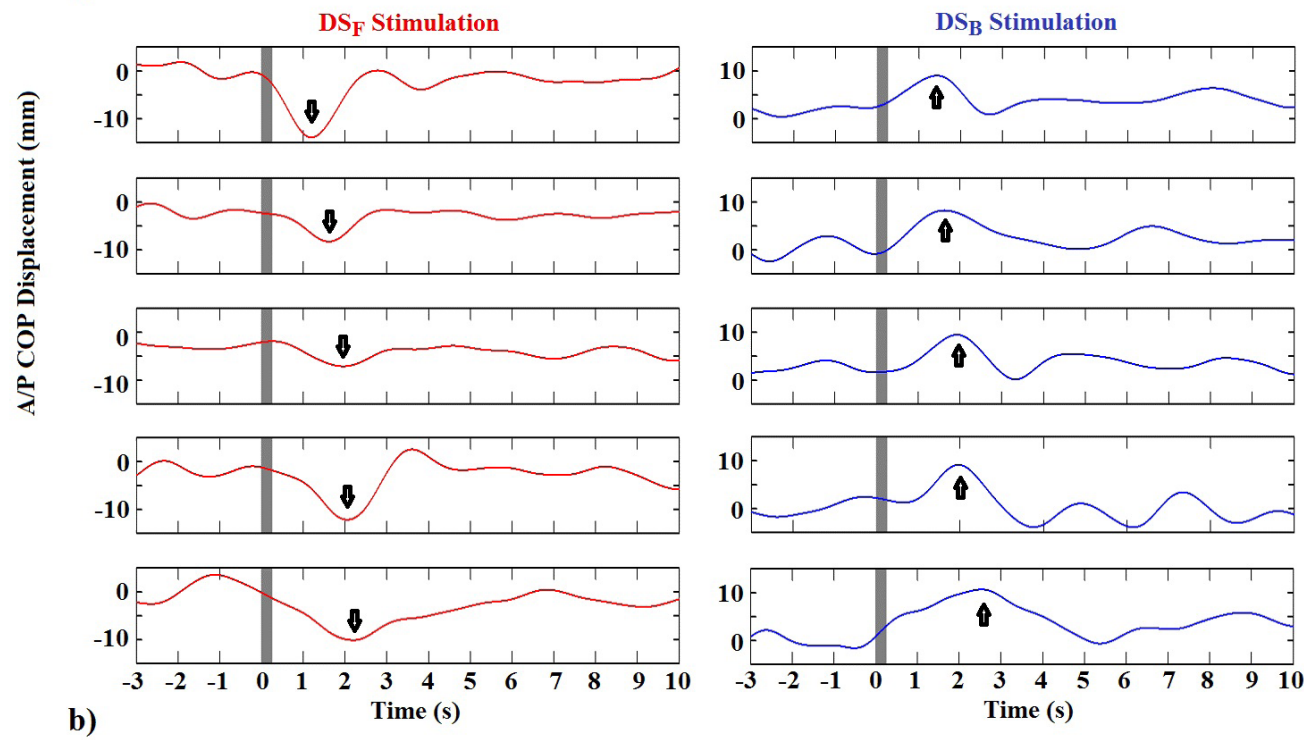

Figure 2. Time evolution of the A/P COP displacement segments during: (a) white wall condition (WW); (b) forward (DS, red) and backward $\left(\mathrm{DS}_{\mathrm{B}}\right.$, blue) virtual scene stimulation. The gray area indicates the $250 \mathrm{~ms}$ of DS (motion-onset: $t=0$ ). The arrows indicate the significant changes (Tukey post-hoc test, $\mathrm{p}<0.001$ ) of the $\mathrm{A} / \mathrm{P}$ COP position in response to egomotion. 
egomotion with evoked postural response in the same direction of the motion scene. Furthermore, the effect of the linear egomotion during $\mathrm{DS}_{\mathrm{F}}$ achieves its peak approximately $300 \mathrm{~ms}$ after $\mathrm{DS}_{\mathrm{B}}$ (gray area in Figure 3 ). Additionally, the subject reestablished the postural stability until the $7^{\text {th }}$ second after DS. Similar results were observed for the rest of the subjects.

Figure 4a depicts the grand-average (29 subjects) of the $\mathrm{A} / \mathrm{P}$ COP displacement for $\mathrm{DS}_{\mathrm{F}}$ (red) and $\mathrm{DS}_{\mathrm{B}}$ (blue) in absolute values. In both DS conditions, the COP mean displacement and its standard deviation increased with the sequence of stimulation (ranging from $8.4 \pm 1.7$ to $22.6 \pm 5.3 \mathrm{~mm}$ ). In addition, the time to recover stability also increased with the sequence of stimulation (Figure $4 \mathrm{~b}$ ), ranging from $4.1 \pm 0.4$ to $7.2 \pm 0.6 \mathrm{~s}$. Although all subjects reported vection, the percentage of the amount of egomotion differs among volunteers, ( $63 \%$ to $80 \%$ of DS trials). Moreover, no difference (Wilcoxon rank sum test: $p=0.7$ ) was observed between COP displacement response distributions due to $\mathrm{DS}_{\mathrm{F}}$ and $\mathrm{DS}_{\mathrm{B}}$ (absolute values), as well as between time to recover stability ( $p=0.8$; Figure 5). On the other hand, the median of the instant peak time distribution of egomotion during $\mathrm{DS}_{\mathrm{F}}$ was different than that obtained for $\mathrm{DS}_{\mathrm{B}}$ ( $p=0.002)$, occurring $200 \mathrm{~ms}$ after $\mathrm{DS}_{\mathrm{B}}$.

\section{Discussion}

In this dynamic virtual protocol, the moving room paradigm was applied with the environmental structures composing an optic flow as a tunnel pattern to generate

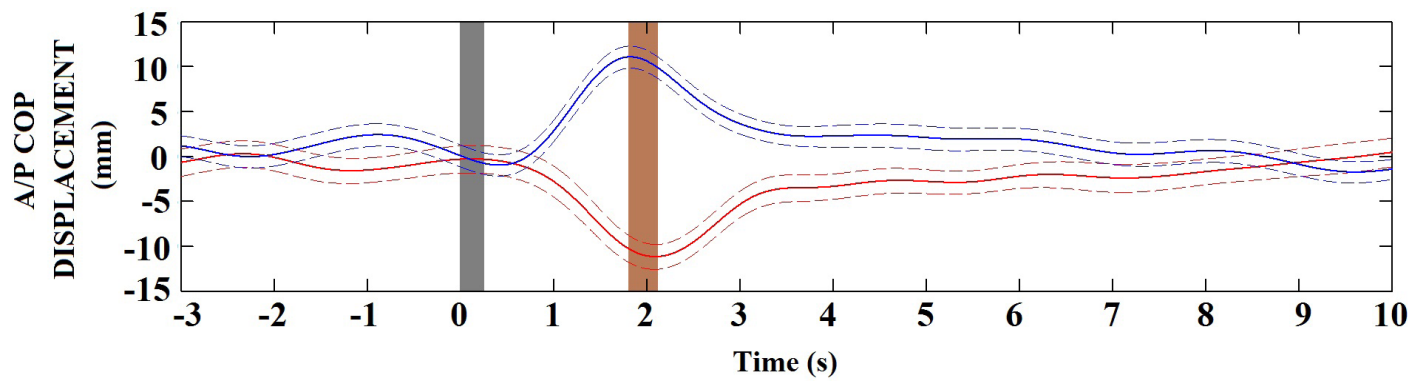

Figure 3. The coherent averaged A/P COP displacement from subject \#1 in response to egomotion induced by forward (DS $\left(\mathrm{DS}_{\mathrm{B}}\right.$, blue) scene stimulation. The dashed lines indicate the standard deviation of the A/P COP displacement. The gray area indicates DS exhibition $(250 \mathrm{~ms})$. The brown area indicates the latency $(300 \mathrm{~ms})$ between evoked postural response by applying $\mathrm{DS}_{\mathrm{F}}$ and $\mathrm{DS}_{\mathrm{B}}$.

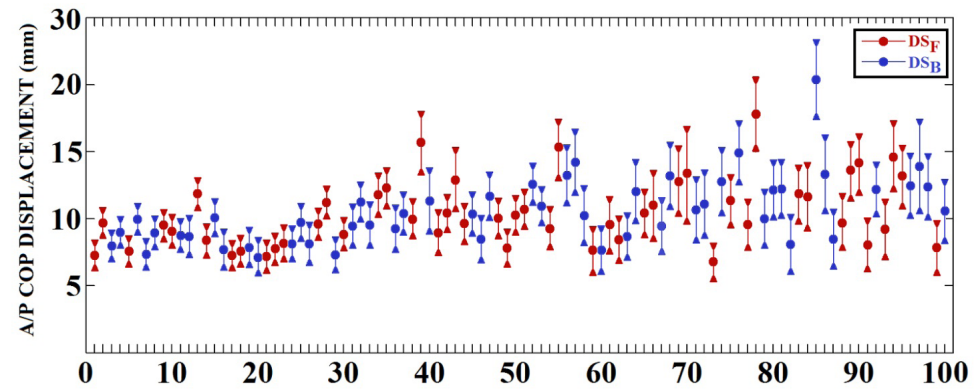

a)

STIMULATING SEQUENCE (\#)

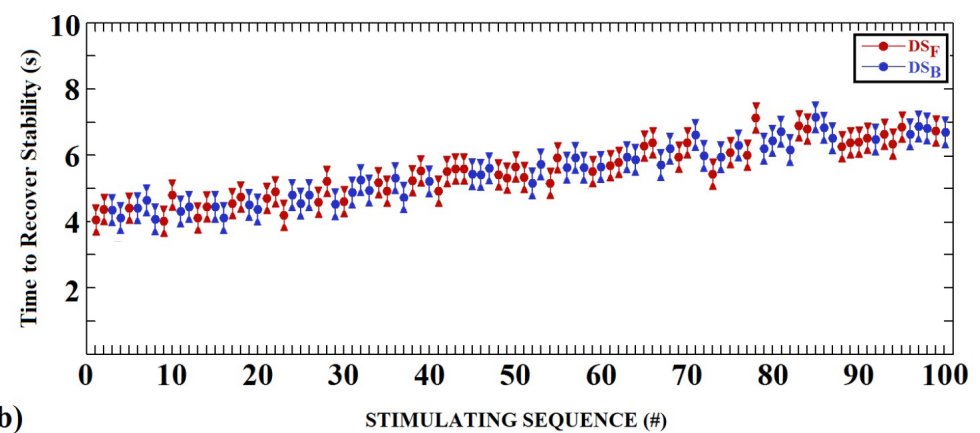

Figure 4. The grand-average over DS trials (mean \pm standard deviation): (a) A/P COP displacement (absolute values); (b) time to recover stability. Red and blue indicates the $\mathrm{DS}_{\mathrm{F}}$ and $\mathrm{DS}_{\mathrm{B}}$, respectively. 
COP DISPLACEMENT

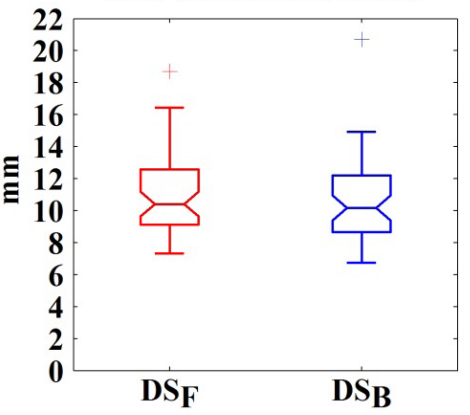

Time to Recover Stability

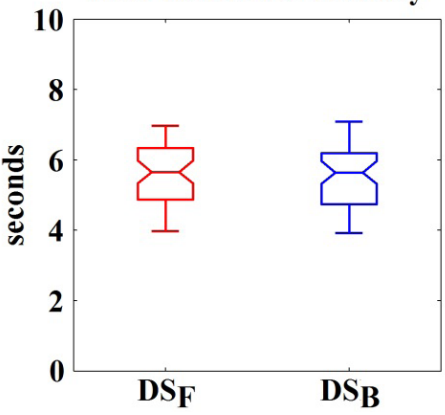

DS PEAK TIME

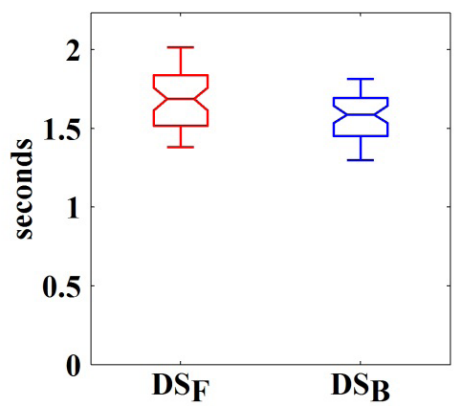

Figure 5. Box-plot distribution of A/P COP displacement (absolute values), time to recover stability and instant peak time of egomotion during $\mathrm{DS}_{\mathrm{F}}$ (red) and $\mathrm{DS}_{\mathrm{B}}$ (blue).

discrete forward or backward sway stimulation. This tunnel pattern induced linear egomotion in the stationary observer, changing the center of pressure position, as also observed by Akizuki et al. (2005), Dokka et al. (2009), Pretto et al. (2009), Streepey et al. (2007) and Wang et al. (2010) during oscillating room stimulation. The virtual scene was designed with an $8.0 \mathrm{~m}$ wide by $3.0 \mathrm{~m}$ high by $12.0 \mathrm{~m}$ deep room in order to stimulate the peripheral vision while the floor moved parallel to a ground plane. It was projected with dimension $1.72 \times 1.16 \mathrm{~m}$ in a white wall located $1 \mathrm{~m}$ ahead from the standing subject $\left(81.4^{\circ} \times 60.2^{\circ}\right.$ visual incidence angle). The size of the lateral view of the present protocol increased the peripheral vision stimulation since it is greater than those used in most virtual reality displays as head-mounted display, shutter glasses or computer monitor (ranging from 30 to $60^{\circ}$ ). According to Pretto et al. (2009), the optic flow carried out by those displays restricts the influence of visual inputs at central visual field. Therefore, the visual incidence angle used in the proposed virtual stimulation protocol establishes the representation of the spatial structure of the employed environment to stimulate the peripheral vision as suggested by Guerraz and Bronstein (2008), O'Connor et al. (2008) and Pretto et al. (2009).

The optic flow parallel to the $\mathrm{A} / \mathrm{P}$ axis of the human body was generated by the translation of the floor, as the classical study of locomotion control by Gibson $(1950 ; 1954)$. This source of information parallel to the $\mathrm{A} / \mathrm{P}$ axis of the human body has been used in most postural control protocols as described by Dokka et al. (2009), O'Connor et al. (2008), Pretto et al. (2009), Seno et al. (2010) and Wang et al. (2010). Distinct of those studies, the reversing black and white background during chessboard-like floor translation applied simultaneously with the lateral walls and furniture movements has accomplished to increase the bi-dimensional optic flow up to $4 \%$ s at the periphery of visual field. Indeed, this optic flow field velocity reflects the natural vision when the virtual scene moves at the same velocity as the physiological body sway perturbation (Berthoz et al., 1975; 1979; Masson et al., 1995).

In the proposed protocol, the luminance was changed by steps of $2 \mathrm{~cd} / \mathrm{m}^{2}$, ranging from 31 to $39 \mathrm{~cd} / \mathrm{m}^{2}$ and increasing (or decreasing) in forward (or backward) direction while the scene is moved with constant velocity $(V i=2.0 \mathrm{~m} / \mathrm{s})$. These luminance thresholds applied here are in the range of those for real image motion detection by the visual system, as reported by Berthoz et al. (1975; 1979); Masson et al. (1995); Pretto et al. (2009). However, these authors used a constant luminance during forward displacement to induce peripheral optic flow information for navigation control, without objects in the central visual field. On the other hand, in order to reproduce the natural optical flow information for the orthostatic posture control, the expansion / reduction of the size of the table and chair in the central visual field was employed. Besides, it was used also to highlight the fusion between the black and white moving pattern of the chessboard-like floor, increasing self-motion sensation and hence the linear egomotion.

Such dynamic visual effect is equivalent to the direction of body motion, i.e. body sway in the anterior axes increases the optic flow of the environment, otherwise (posterior sway) the optic flow decreases. As a consequence, the proposed protocol induced the illusion of self-motion in opposite direction of the DS scene, as observed in the literature (Berthoz et al., 1975; Dijkstra et al., 1994; Dokka et al., 2009; Gibson, 1950; Keshner and Kenyon, 2000; Lestienne et al., 1977; Masson et al., 1995; O’Connor et al., 2008; Paulus et al., 1989; Pretto et al., 2009; Seno et al., 2009; 2010; Stoffregen, 1985; 1986; Van Asten et al., 1988; Wang et al., 2010). Therefore, the bi-dimensional optic flow reflects the 3D layout of this virtual environment available to the stationary observer, representing the magnitude, velocity and direction of body sway displacement. Moreover, the proposed setup allows 
including additional furniture, change the scene velocity or the amplitude of displacement, as well as roll the surrounding. However, these can be properly evaluated in future studies in order to provide evidence that the system can be useful in a broad set of postural control experiments. Hence, such flexibility allows investigating the interaction (or conflict) between the visual system and the proprioception or vestibular system without mechanic devices (as platform tilt, translating sled or roll chair), generally used in most studies. On the other hand, such devices cannot be fully discarded, since the direct stimulation of the other systems provides specific information, which cannot be generated by a visual stimulation system.

The forwards and backwards scenes were applied in random order and hence the subject could not predict the direction of surrounding motion. Additionally, the dynamic virtual stimulation with unexpected visual motion-onset distracter scenes was used to avoid postural adaptation or optic flow habituation, as suggested by O'Connor et al. (2008), Reed-Jones et al. (2008) and Wang et al. (2010). These authors reported that consecutive visual scene motion at the same direction decreases linear egomotion in about $40 \%$ and can raise it in $10 \%$ after 10 stimuli. Although Reed-Jones et al. (2008) reported that the A/P COP sway displacement decreases with the repetition of the presentation, this was not observed in our study. Indeed, the unexpected surrounding motion creates a mismatch between the dynamic virtual scene and motion of the body. According to Wang et al. (2010), this divergence produces a sensory conflict that interferes with the capability to discriminate between visual field motion and body motion, resulting in spatial disorientation and changing the center of pressure position.

Linear egomotion was observed by applying $250 \mathrm{~ms}$ of a dynamic scene with a constant velocity of $2 \mathrm{~m} / \mathrm{s}$ and interspersed by $10 \mathrm{~s}$ of static scene at the final position of the exhibiting room movement. The orthostatic postural response occurred at the same direction of the dynamic stimulation, with A/P COP displacement and its standard deviation increasing with the random sequence of stimulation. The results agree with the literature: self-motion in scene velocity greater than $1 \mathrm{~m} / \mathrm{s}$ and sway amplitude increasing at scene velocities as high as $2.0 \mathrm{~m} / \mathrm{s}$ (Akizuki et al., 2005; Darekar et al., 2015; Dokka et al., 2009; 2010; Keshner and Kenyon, 2004; Streepey et al., 2007; Wang et al., 2010). Those studies have demonstrated that vection and postural response are correlated with the velocity of the visual motion stimulation. Dokka et al. $(2009 ; 2010)$ observed body sway at scene velocity of 1.25 and $1.88 \mathrm{~m} / \mathrm{s}$ when compared with lower scene velocities $(<0.31 \mathrm{~m} / \mathrm{s})$. Additionally, the visually evoked postural responses was lined up with the plane of motion of the visual stimulus, while the magnitude of body displacement increases during linear egomotion when compared to the static scenes, also observed by Akizuki et al. (2005), Da-Silva et al., 2015; Dijkstra et al. (1992; 1994) and Wang et al. (2010).

The proposed dynamic virtual protocol induced linear egomotion in more than $63 \%$ of the applied trials. Considering that the subject reestablishes the postural stability until the $7^{\text {th }}$ second, the $10 \mathrm{~s}$ of the static scene interval interspersed between the dynamic stimulation ensured that, when a new dynamic stimulus was applied, no significant effect was observed from the previous one (ANOVA, $p>0.8$ ). Therefore, the postural instability is also a time-locked activity (Da-Silva et al., 2015). This finding indicates the influence of the static scene to recover stability after the body sway perturbation. According to Dijkstra et al. (1992; 1994), Fushiki et al. (2005), Haibach et al., 2008 and Seno et al. (2010), the static scene provides a time to recover stability during the action-perception cycle for postural control in a moving visual environment, avoiding the risk of fall. On the other hand, the increasing of the $\mathrm{A} / \mathrm{P}$ $\mathrm{COP}$ and time to recover stability variability along sequence of stimulation could be associated to the prolonged upright stance during the protocol applied here. To reduce this variability, we recommend for further study that the amount of the dynamic visual stimulation can be applied into distinct blocks, with subject resting in a comfortable chair between blocks.

The results indicate that $\mathrm{DS}_{\mathrm{F}}$ peak occurs at approximately $200 \mathrm{~ms}$ after $\mathrm{DS}_{\mathrm{B}}$ peak. This finding could be an indicative that the effect due to forward stimulation elicits distinct control strategies to maintained balance than that observed by applying the backward stimulation. This difference perhaps could represents a higher order cortical processing to maintaining balance, since there are more magnocellular receptive fields regarding to the increasing size of the image at the retina, as well as the motion-processing pathway that can be traced from those magnocellular input to primary visual cortex and allied areas of the parietal cortex (Kandel, 1991; Kuba et al., 2007). Hence, the postural control strategy to maintain balance seems to depend on the virtual scene direction.

All these findings indicate that linear egomotion induced by forward and backward visual motion stimulation was a major feature to promote $\mathrm{A} / \mathrm{P}$ postural instability. Besides, it is in agreement with the physiological properties of the visual system: the expansion (or reduction) of the scene increases (or decreases) the size of the image at the retina and 
the optic flow in the periphery of the visual field (Paulus et al., 1989; Seno et al., 2009; Stoffregen, 1985). Thus, it evokes an illusion that something moves towards (or backwards) the subject, as pointed out by Berthoz et al. (1975; 1979), O'Connor et al. (2008), Pretto et al. (2009), Reed-Jones et al. (2008) and Wang et al. (2010). This finding suggests that linear egomotion and the direction of the A/P COP displacement during distinct dynamic scenes are directly associated, as reported by Fushiki et al. (2005) and Wang et al. (2010). Therefore, the virtual dynamic visually evoked postural responses during stabilometric test cannot be elicited without an illusion of egomotion.

Hence, the dynamic property of the proposed protocol generates visual optic flow stimulation as a tunnel pattern similar to the natural vision during orthostatic sway. The virtual stimulation induces COP displacements response due to dynamic layout available to a stationary observer. The effects of linear egomotion are dependent on the direction of the dynamic visual stimulation. This finding indicates the potential application of the proposed virtual dynamic visual stimulation protocol to investigate the cortical visual evoked response in postural control studies.

\section{Acknowledgements}

Special thanks to our Professor Antonio Fernando Catelli Infantosi (in memorian) for dedication and expertise in Biomedical Engineering. Professor Antonio Infantosi will always be our mentor. Thanks to our best friend, João Gama (in memorian), for his dedication and for the books provided, enabling our improvement in the art of biological signal processing and neural engineering. The authors are thankful to all participants for investing their time and effort. This research was financially supported by FAPERJ and the Brazilian Research Council (CNPq).

\section{References}

Akizuki H, Uno A, Arai K, Morioka S, Ohyama S, Nishiike S, Tamura K, Takeda N. Effects of immersion in virtual reality on postural control. Neuroscience Letters. 2005; 379(1):23-6. http://dx.doi.org/10.1016/j.neulet.2004.12.041. PMid:15814192.

Andersen GJ, Dyre BP. Spatial orientation from optic flow in the central visual field. Perception \& Psychophysics. 1989; 45(5):453-8. http://dx.doi.org/10.3758/BF03210719. PMid:2726408.

Berthoz A, Lacour M, Soechting JF, Vidal PP. The role of vision in the control of posture during linear motion. Progress in Brain Research. 1979; 50:197-209. http:// dx.doi.org/10.1016/S0079-6123(08)60820-1. PMid:551426.
Berthoz A, Pavard B, Young LR. Perception of linear horizontal self-motion induced by peripheral vision (linearvection): basic characteristics and visual-vestibular interactions. Experimental Brain Research. 1975; 23(5):471-89. http:// dx.doi.org/10.1007/BF00234916. PMid:1081949.

Da Silva PJG, Nadal J, Infantosi AFC. Investigating the center of pressure velocity Romberg's quotient for assessing the visual role on the body sway. Revista Brasileira de Engenharia Biomédica. 2012; 28(4):319-26. http://dx.doi. org/10.4322/rbeb.2012.039.

Darekar A, McFadyen BJ, Lamontagne A, Fung J. Efficacy of virtual reality-based intervention on balance and mobility disorders post-stroke: a scoping review. Journal of Neuroengineering and Rehabilitation. 2015; 12(1):46. http:// dx.doi.org/10.1186/s12984-015-0035-3. PMid:25957577.

Da-Silva PJG, Cagy M, Infantosi AFC. A new dynamic virtual stimulation protocol to induce vection. IFMBE Proceedings, 2015; 45:497-500. http://dx.doi.org/10.1007/9783-319-11128-5 124.

Day BL, Guerraz M. Feedforward versus feedback modulation of human vestibular-evoked balance responses by visual self-motion information. The Journal of Physiology. 2007; 582(1):153-61. http://dx.doi.org/10.1113/jphysiol.2007.132092. PMid:17446222.

Dijkstra TMH, Gielen CCAM, Melis BJM. Postural responses to stationary and moving scenes as a function of distance to the scene. Human Movement Science. 1992; 11(1-2):195203. http://dx.doi.org/10.1016/0167-9457(92)90060-O.

Dijkstra TMH, Schöner G, Gielen CCAM. Temporal stability of the action-perception cycle for postural control in a moving visual environment. Experimental Brain Research. 1994; 97(3):477-86. http://dx.doi.org/10.1007/BF00241542. PMid:8187859.

Dokka K, Kenyon RV, Keshner EA, Kording KP. Self versus environment motion in postural control. PLoS Computational Biology. 2010; 6(2):1-8. http://dx.doi.org/10.1371/journal. pcbi.1000680. PMid:20174552.

Dokka K, Kenyon RV, Keshner EA. Influence of visual scene velocity on segmental kinematics during stance. Gait \& Posture. 2009; 30(2):211-6. http://dx.doi.org/10.1016/j. gaitpost.2009.05.001. PMid:19505827.

Fushiki H, Kobayashi K, Asai M, Watanabe Y. Influence of visually induced self-motion on postural stability. Acta Oto-Laryngologica. 2005; 125(1):60-4. http://dx.doi. org/10.1080/00016480410015794. PMid:15799576.

Gibson JJ. The perception of the visual world. Oxford: Houghton Mifflin; 1950.

Gibson JJ. The visual perception of objective motion and subjective movement. Psychological Review. 1954; 61(5):30414. http://dx.doi.org/10.1037/h0061885. PMid:13204493.

Guerraz M, Bronstein AM. Mechanisms underlying visually induced body sway. Neuroscience Letters. 2008; 443(1):12-6. http://dx.doi.org/10.1016/j.neulet.2008.07.053. PMid:18672020.

Guerraz M, Gianna CC, Burchill PM, Gresty MA, Bronstein AM. Effect of visual surrounding motion on body sway in a 
three-dimensional environment. Perception \& Psychophysics. 2001a; 63(1):47-58. http://dx.doi.org/10.3758/BF03200502. PMid:11304016.

Guerraz M, Thilo KV, Bronstein AM, Gresty MA. Influence of action and expectation on visual control of posture. Brain Research. Cognitive Brain Research. 2001b; 11(2):25966. http://dx.doi.org/10.1016/S0926-6410(00)00080-X. PMid:11275487.

Haibach PS, Slobounov SM, Newell KM. The potential applications of a virtual moving environment for assessing falls in elderly adults. Gait \& Posture. 2008; 27(2):303-8. http:// dx.doi.org/10.1016/j.gaitpost.2007.04.004. PMid:17524647.

Kandel ER. Perception of motion, depth, and form. In: Kandel ER, Schwartz JH, Jessel TM, editors. Principles of neurological science. New York: Elsevier; 1991. p. 440-66.

Keshner EA, Dokka K, Kenyon RV. Influences of the perception of self-motion on postural parameters. Cyberpsychology \& Behavior. 2006; 9(2):163-6. http://dx.doi.org/10.1089/ cpb.2006.9.163. PMid:16640471.

Keshner EA, Kenyon RV, Langston J. Postural responses exhibit multisensory dependencies with discordant visual and support surface motion. Journal of Vestibular Research. 2004; 14(4):307-19. PMid:15328445.

Keshner EA, Kenyon RV. The influence of an immersive virtual environment on the segmental organization of postural stabilizing responses. Journal of Vestibular Research. 2000; 10(4-5):201-19.

Keshner EA, Kenyon RV. Using immersive technology for postural research and rehabilitation. Assistive Technology. 2004; 16(1):54-62. http://dx.doi.org/10.1080/10400435.20 04.10132074. PMid:15357148.

Kuba M, Kubová Z, Kremlácek J, Langrová J. Motion-onset VEPs: Characteristics, methods, and diagnostic use. Vision Research. 2007; 47(2):189-202. http://dx.doi.org/10.1016/j. visres.2006.09.020. PMid:17129593.

Kuno S, Kawakita T, Kawakami O, Miyake Y, Watanabe S. Postural adjustment response to depth direction moving patterns produced by virtual reality graphics. Japanese Journal of Physiology. 1999; 49(5):417-24. http://dx.doi. org/10.2170/jjphysiol.49.417. PMid:10603425.

Lestienne F, Soechting J, Berthoz A. Postural readjustments induced by linear motion of visual scenes. Experimental Brain Research. 1977; 28(3-4):363-84. PMid:885185.

Lishman JR, Lee DN. The autonomy of visual kinaesthesis. Perception. 1973; 2(3):287-94. http://dx.doi.org/10.1068/ p020287. PMid:4546578.

Masson G, Mestre DR, Blin O, Pailhous J. Low luminance contrast sensitivity: effect of training on psychophysical and optokinetic nystagmus thresholds in man. Vision Research. 1994; 34(14):1893-9. http://dx.doi.org/10.1016/00426989(94)90313-1. PMid:7941391.

Masson G, Mestre DR, Pailhous J. Effects of the spatiotemporal structure of optical flow on postural readjustments in man. Experimental Brain Research. 1995; 103(1):137-50. http://dx.doi.org/10.1007/BF00241971. PMid:7615029.
Mestre DR. Visual perception of self-motion. In: Proteau L, Elliott D, editors. Vision and motor control. Amsterdam: Elsevier Science Publishers B.V.; 1992. p. 421-38.

O’Connor KW, Loughlin PJ, Redfern MS, Sparto PJ. Postural adaptations to repeated optic flow stimulation in older adults. Gait \& Posture. 2008; 28(3):385-91. http:// dx.doi.org/10.1016/j.gaitpost.2008.01.010. PMid:18329878.

Paulus W, Straube A, Brandt T. Visual stabilization of posture: physiological stimulus characteristics and clinical aspects. Brain. 1984; 107(Pt 4):1143-63. http://dx.doi.org/10.1093/ brain/107.4.1143. PMid:6509312.

Paulus W, Straube A, Krafczyk S, Brandt T. Differential effects of retinal target displacement, changing size and changing disparity in the control of anterior/posterior and lateral body-sway. Experimental Brain Research. 1989; 78(2):243-52. http://dx.doi.org/10.1007/BF00228896. PMid:2599035.

Pretto P, Ogier M, Bülthoff HH, Bresciani JP. Influence of size of the field of view on motion perception. Computer Graphics. 2009; 33(2):139-46. http://dx.doi.org/10.1016/j. cag.2009.01.003.

Reed-Jones RJ, Vallis LA, Reed-Jones JG, Trick LM. The relationship between postural stability and virtual environment adaptation. Neuroscience Letters. 2008; 435(3):204-9. http:// dx.doi.org/10.1016/j.neulet.2008.02.047. PMid:18359162.

Seno T, Ito H, Sunaga S. The object and background hypothesis for vection. Vision Research. 2009; 49(24):2973-82. http:// dx.doi.org/10.1016/j.visres.2009.09.017. PMid:19782099.

Seno T, Nakamura S, Ito H, Sunaga S. Static visual components without depth modulation alter the strength of vection. Vision Research. 2010; 50(19):1972-81. http:// dx.doi.org/10.1016/j.visres.2010.07.004. PMid:20633575.

Slobounov S, Wu T, Hallett M, Shibasaki H, Slobounov E, Newell K. Neural underpinning of postural responses to visual field motion. Biological Psychology. 2006; 72(2):18897. http://dx.doi.org/10.1016/j.biopsycho.2005.10.005. PMid: 16338048

Stoffregen TA. Flow structure versus retinal location in the optical control of stance. Journal of Experimental Psychology. Human Perception and Performance. 1985; 11(5):554-65. http://dx.doi.org/10.1037/0096-1523.11.5.554. PMid:2932530.

Stoffregen TA. The role of optical velocity in the control of stance. Perception \& Psychophysics. 1986; 39(5):355-60. http://dx.doi.org/10.3758/BF03203004. PMid:3737368.

Streepey JW, Kenyon RV, Keshner EA. Field of view and base of support width influence postural responses to visual stimuli during quiet stance. Gait \& Posture. 2007; 25(1):49-55. http://dx.doi.org/10.1016/j.gaitpost.2005.12.013. PMid:16464594.

Tossavainen T, Juhola M, Pyykkö I, Aalto H, Toppila E. Development of virtual reality stimuli for force platform posturography. International Journal of Medical Informatics. 2003; 70(2-3):277-83. http://dx.doi.org/10.1016/S13865056(03)00034-0. PMid:12909179. 
Van Asten WNJC, Gielen CCAM, Van der Gon JJD. Postural adjustments induced by simulated motion of differently structured environments. Experimental Brain Research. 1988; 73(2):371-83. http://dx.doi.org/10.1007/BF00248230. PMid:3215313.

Wang Y, Kenyon RV, Keshner EA. Identifying the control of physically and perceptually evoked sway responses with coincident visual scene velocities and tilt of the base of support.
Experimental Brain Research. 2010; 201(4):663-72. http:// dx.doi.org/10.1007/s00221-009-2082-0. PMid:19924408.

Warren WH Jr, Hannon DJ. Direction of self-motion is perceived from optical flow. Nature. 1988; 336(6195):162-3. http://dx.doi.org/10.1038/336162a0.

Warren WH. Self-motion: visual perception and visual control. In: Epstein W, Rogers S, editors. Perception of space and motion. London: Academic Press; 1995. p. 263-325.

\footnotetext{
Authors

Paulo José Guimarães Da-Silva ${ }^{1 *}$, Maurício Cagy ${ }^{1}$, Antonio Fernando Catelli Infantosi ${ }^{1}$

${ }^{1}$ Biomedical Engineering Program, Centro de Tecnologia, Instituto Alberto Luiz Coimbra de Pós-graduação e Pesquisa de Engenharia - COPPE, Universidade Federal do Rio de Janeiro - UFRJ, Av. Horácio Macedo, 2030, B1. H, Sala 327, Cidade Universitária, CEP 21941-914, Rio de Janeiro, RJ, Brazil.
} 